\title{
Assessing Chemical-Induced Liver Injury In Vivo From In Vitro Gene Expression Data in the Rat: The Case of Thioacetamide Toxicity
}

\section{OPEN ACCESS}

\section{Edited by:}

Joao Batista Teixeira da Rocha, Universidade Federal de Santa Maria,

Brazil

Reviewed by:

Suzy Munir Salama

University of Malaya, Malaysia

Sudin Bhattacharya,

Michigan State University, United States

*Correspondence:

Patric Schyman

pschyman@bhsai.org

Masakazu Shiota

masakazu.shiota@vanderbilt.edu

tThese authors have contributed equally to this work

Specialty section:

This article was submitted to

Toxicogenomics,

a section of the journal

Frontiers in Genetics

Received: 05 March 2019 Accepted: 06 November 2019

Published: 26 November 2019

Citation:

Schyman P, Printz RL, Estes SK, O'Brien TP, Shiota M and Wallqvist A (2019) Assessing Chemical-Induced Liver Injury In Vivo From In Vitro Gene Expression Data in the Rat: The Case

of Thioacetamide Toxicity.

Front. Genet. 10:1233.

doi: 10.3389/fgene.2019.01233

\author{
Patric Schyman ${ }^{1,2 * t}$, Richard L. Printz ${ }^{3+}$, Shanea K. Estes ${ }^{3}$, Tracy P. O’Brien ${ }^{3}$, \\ Masakazu Shiota ${ }^{3 *}$ and Anders Wallqvist ${ }^{1}$
}

${ }^{1}$ DoD Biotechnology High Performance Computing Software Applications Institute, Telemedicine and Advanced Technology Research Center, U.S. Army Medical Research and Development Command, Fort Detrick, MD, United States, ${ }^{2}$ The Henry M. Jackson Foundation for the Advancement of Military Medicine Inc. (HJF), Bethesda, MD, United States, ${ }^{3}$ Department of Molecular Physiology and Biophysics, Vanderbilt University School of Medicine, Nashville, TN, United States

Consumers are exposed to thousands of chemicals with potentially adverse health effects. However, these chemicals will never be tested for toxicity because of the immense resources needed for animal-based (in vivo) toxicological studies. Today, there are no viable in vitro alternatives to these types of animal studies. To develop an in vitro approach, we investigated whether we could predict in vivo organ injuries in rats with the use of RNA-seq data acquired from tissues early in the development of toxicant-induced injury, by comparing gene expression data from RNA isolated from these rat tissues with those obtained from in vitro exposure of primary liver and kidney cells. We collected RNA-seq data from the liver and kidney tissues of Sprague-Dawley rats 8 or $24 \mathrm{~h}$ after exposing them to vehicle (control), low $(25 \mathrm{mg} / \mathrm{kg})$, or high $(100 \mathrm{mg} / \mathrm{kg})$ doses of thioacetamide, a known liver toxicant that promotes fibrosis; we used these doses and exposure times to cause only mild toxicantinduced injury. For the in vitro study, we treated two cell types from Sprague-Dawley rats, primary hepatocytes (vehicle; low, $0.025 \mathrm{mM}$; or high, $0.125 \mathrm{mM}$ dose), and renal tube epithelial cells (vehicle; low, $0.125 \mathrm{mM}$; or high, $0.500 \mathrm{mM}$ ) dose) with the thioacetamide metabolite, thioacetamide-S-oxide, selecting in vitro doses and exposure times to recreate the early-stage toxicant-induced injury model that we achieved in vivo. RNA-seq data were collected 9 or $24 \mathrm{~h}$ after application of vehicle or thioacetamide-S-oxide. We found that our modular approach for the analysis of gene expression data derived from in vivo RNAseq strongly correlated $\left(R^{2}>0.6\right)$ with the in vitro results at two different dose levels of thioacetamide/thioacetamide-S-oxide after $24 \mathrm{~h}$ of exposure. The top-ranked liver injury modules in vitro correctly identified the ensuing development of liver fibrosis.

Keywords: predictive toxicology, RNA-seq, thioacetamide, toxicogenomics, fibrosis, in vitro-in vivo correlations 


\section{INTRODUCTION}

Thioacetamide was developed as an effective pesticide to control the decay of citrus fruits (Childs and Siegler, 1945), but was soon found to cause liver diseases (fibrosis and cirrhosis) and liver tumors (Fitzhugh and Nelson, 1948). It has been used extensively in animal studies (Ledda-Columbano et al., 1991; Li et al., 2002; Yeh et al., 2004; Okuyama et al., 2005; Dwivedi and Jena, 2018), largely for its ability to cause acute liver damage (Li et al., 2002; Okuyama et al., 2005). As consumers, we are exposed to thousands of chemicals with potentially adverse health effects. Yet, many of these chemicals will never be tested for toxicity as extensively as thioacetamide because of the immense resources needed for animal-based (in vivo) toxicological studies. There is also an ethical aspect to conducting large-scale animal experiments, which must meet legal and regulatory requirements, as directed by the Animal Welfare Act (AWA) in the U.S. and the European Directive 2010/63/EU to implement the principles of replacement, reduction, and refinement of the use of animals in research. Consequently, major efforts are currently under way to develop non-animal-based testing methods, such as high-throughput cell-based (in vitro) assays (Dix et al., 2007; Adeleye et al., 2015; Goh et al., 2015; Wetmore, 2015). However, these platforms are unlikely to meet the requirements for safety assessment and replace animal testing until reliable in vivo-in vitro correlations are achieved (Wetmore, 2015). Therefore, we need an effective approach to link in vitro results to meaningful in vivo injury endpoints, such as liver fibrosis.

Here, we present our efforts to use a systems toxicology approach to address the discrepancies commonly found between in vitro and in vivo results. We focus on toxicogenomics, a subfield of toxicology, which assumes that toxicity is accompanied by a change in the expression of either a single gene or a set of genes (Hamadeh et al., 2002; Sutherland et al., 2017), and that chemical exposures leading to the same injury endpoint cause similar changes in gene expression. Toxicogenomics has advanced the understanding of toxicological effects and improved predictions of responses to chemicals (Steiner et al., 2004; Sutherland et al., 2017). Several toxicogenomic approaches use single genes or coexpressed genes to study stress responses. For example, when specific genes are associated with an injury (e.g., cancer, cholestasis, steatosis), gene signatures are often used to classify chemicals in terms of their toxicity endpoints (Segal et al., 2004; Sahini et al., 2014; Parmentier et al., 2017). When sets of genes are differentially activated in response to an injury condition, such gene sets are often referred to as toxicity pathways or coexpressed genes. Furthermore, data mining techniques, such as bi-clustering (Pontes et al., 2015), are used to create sets of coexpressed genes, which consist of genes whose expression pattern is correlated across a set of chemical exposures with a common injury endpoint (Sutherland et al., 2017).

Previously, we developed an unbiased protocol to assign sets of coexpressed genes (modules) associated with molecular toxicity and linked them to specific injuries in the liver and kidney, using the Iterative Signature Algorithm (Bergmann et al., 2003; Tawa et al., 2014; AbdulHameed et al., 2016; Te et al., 2016). Compared to gene signatures, coexpressed gene modules can make more robust predictions for specific pathologies because they rely on groups of genes rather than individual genes. Gene expression data are prone to inherent noise, owing to limitations in experimental techniques and the complexity of biological systems. A limitation of the gene module approach is that the modules do not contain the same mechanistic information as do biological pathways, where genes can be linked by their function. Using gene expression data from the Open Toxicogenomics Project-Genomics Assisted Toxicity Evaluation System (TG-GATEs) database, which contains data from Sprague-Dawley rats exposed to different chemicals for 4 to 29 days (Igarashi et al., 2015), we derived 8 and 11 chemical-induced injury modules for the liver and kidney, respectively, associated with the relevant histopathological injury phenotypes from the TG-GATEs database (Te et al., 2016). Recently, we validated our injury modules in vivo by exposing Sprague-Dawley rats to a low $(25 \mathrm{mg} / \mathrm{kg})$ or high $(100 \mathrm{mg} / \mathrm{kg})$ dose of thioacetamide for 8 or 24 $\mathrm{h}$ (Schyman et al., 2018). The most activated injury modules were those associated with cellular infiltration and fibrosis, consistent with previous studies on thioacetamide toxicity and suggested by our own histological analyses.

Our aim in this study was to test the hypothesis that injury modules identified from in vitro transcriptomic responses can correlate with injury modules from in vivo to assist in the prediction of in vivo injury endpoints. For the in vitro-in vivo comparison, we selected thioacetamide as a toxicant for its ability to cause acute liver damage (Li et al., 2002; Okuyama et al., 2005). Thioacetamide is highly toxic to the liver in vivo because it is rapidly metabolized by cytochrome $\mathrm{P} 450$ and flavin-containing monooxygenases into its reactive metabolites (thioacetamide-Soxide and reactive oxygen species) (Hajovsky et al., 2012). In our in vitro studies, we used the metabolized form of the compound, thioacetamide-S-oxide (Hajovsky et al., 2012), to simulate the level of toxicity achieved in vivo after exposure to thioacetamide. To compare liver- and kidney-specific responses, we treated two types of primary cells from Sprague-Dawley rats, hepatocytes, and renal tube epithelial cells, with vehicle (control) or two different doses of thioacetamide-S-oxide (designated low and high), and collected RNA samples at two different time points to ensure a match to an in vivo early-stage injury model. For the in vivo comparisons, we used data from our published thioacetamide toxicity study (Schyman et al., 2018) of 30 Sprague-Dawley rats treated with either vehicle (control) or one of two doses (low; $25 \mathrm{mg} / \mathrm{kg}$ or high; $100 \mathrm{mg} / \mathrm{kg}$ ) of thioacetamide to produce different degrees of early injury. We collected RNA samples for gene expression analysis from the liver and kidney 8 and $24 \mathrm{~h}$ following injection of vehicle or thioacetamide.

\section{MATERIALS AND METHODS}

\section{Experimental Procedures Thioacetamide In Vivo}

Male Sprague-Dawley rats at 10 weeks of age were purchased from Charles River Laboratories (Wilmington, MA). Rats were fed with Formulab Diet 5001 (Purina LabDiet; Purina Miles, Richmond, IN) and given water ad libitum in an environmentally controlled room at a temperature of $23^{\circ} \mathrm{C}$ with 
a 12:12-h light-dark cycle. All experiments were conducted in accordance with the Guide for the Care and Use of laboratory Animals of the U.S. Department of Agriculture, the Vanderbilt University Institutional Animal Care and Use Committee, and USAMRDC Animal Care and Use Review Office. Animals (30 rats) were administered either vehicle (saline; $3 \mathrm{ml} / \mathrm{kg} ; \mathrm{n}=5$ each at two time points) or thioacetamide (25 or $100 \mathrm{mg} / \mathrm{kg}$; $\mathrm{n}=5$ each at two time points for each dose) intraperitoneally at $9 \mathrm{am}$, and the liver and kidney from each animal were harvested 8 or $24 \mathrm{~h}$ after the administration of vehicle or thioacetamide. Rats were anesthetized by intravenous injection of sodium pentobarbital through a jugular vein catheter and then the liver and kidney were dissected and frozen using Wollenberger tongs precooled in liquid nitrogen. The collected plasma, urine, and organs were kept at $-80^{\circ} \mathrm{C}$ until used for analyses. Frozen whole kidneys were powdered in liquid nitrogen, since this organ is histologically heterogeneous. Total RNA was isolated from the liver and powered kidney, using TRIzol Reagent (Thermo Fisher Scientific, Waltham, MA) and the direct-zol RNA MiniPrep kit (Zymo Research, Irvine, CA). We refer the reader to our original publication for further details (Schyman et al., 2018).

\section{Thioacetamide In Vitro}

Cryopreserved rat (Sprague-Dawley) hepatocytes and renal proximal tubular epithelial cells were purchased from Triangle Research Labs (Research Triangle Park, NC) and Sciencell Research Laboratories (Carlsbad, CA), respectively. Hepatocytes were thawed and suspended in thawing medium (MCRT50; Triangle Research Labs) at 6-7 million cells $/ 50 \mathrm{ml}$. The suspension was centrifuged at $50 \times \mathrm{g}$, and cells were resuspended in plating medium (MP100; Triangle Research Labs). Hepatocytes were plated on collagen 1-coated 96-well plates at a density of $2 \times$ $10^{4}$ cells/well for measurement of cell viability and on collagen 1 -coated 6-well plates at a density of $4.5 \times 10^{5}$ cells/well for RNA sequence analysis. Cells were cultured under $5 \% \mathrm{CO}_{2}$ in a $37^{\circ} \mathrm{C}$ incubator. After $4 \mathrm{~h}$ of culture to allow cell attachment, the medium was replaced with hepatocyte maintenance medium (CC-3198, Triangle Research Labs). Rat renal tubular epithelial cells were thawed and suspended in "Epithelial Cell Medium-animal" (EpiCM-a, Sciencell Research Laboratories) and plated into polyL-lysine-coated 96-well plates at a density of $2 \times 10^{4}$ cells/well for measurement of cell viability and on poly-L-lysine-coated 6-well plates at a density of $3 \times 10^{5}$ cells/well for RNA collection. Cells were cultured under $5 \% \mathrm{CO}_{2}$ in a $37^{\circ} \mathrm{C}$ incubator. After $4 \mathrm{~h}$ of culture to allow cell attachment, the medium was replaced by the same medium. Both rat hepatocytes and renal cells were cultured for an additional $18 \mathrm{~h}$ before addition of thioacetamide-S-oxide or vehicle (maintenance medium; CC-3198 for hepatocytes and EpiCM-a for renal cells).

Preliminary studies were performed on rat hepatocytes and renal cells to identify a dose (range: 0.025 to $4 \mathrm{mM}$ for hepatocytes, 0.125 to $4 \mathrm{mM}$ for renal cells) of thioacetamide-S-oxide and length of exposure (range: 3 to $24 \mathrm{~h}$ for both hepatocytes and renal cells) that would result in mild toxicity without substantial loss of cell viability. Two cell viability assays were performed. First, to measure cellular loss of lactate dehydrogenase (LDH), cells were collected and cellular LDH activity remaining after each treatment was measured using the Lactate Dehydrogenase Activity Assay Kit (Sigma-Aldrich, St. Louis, MO). Second, cellular adenosine triphosphate (ATP) levels were measured using the CellTier-Glo 2.0 Assay kit (Promega Co., Madison, WI) according to the manufacturer's protocol. The time-course profiles of cell viablity in these prelimiary studies are illustrated in Figures S1-S4 (Supplemental Material). Based on these studies, two time points, 9 and $24 \mathrm{~h}$, and two doses of thioacetamideS-oxide, designated low and high, were chosen to induce earlystage toxicant-nduced injury with no or little loss in cell viability. Hepatocytes and renal cells were thus exposed for 9 or $24 \mathrm{~h}$ to either vehicle or thioacetamide-S-oxide $(0.025$ or $0.125 \mathrm{mM}$ for hepatocytes, and 0.125 or $0.5 \mathrm{mM}$ for renal cells). Table 1 shows the viability of hepatocytes and renal proximal tubular epithelial cells exposed to thioacetamide for 9 or $24 \mathrm{~h}$ compared to vehicleexposed cells.

Total RNA was isolated from culture cells using TRIzol Reagent (Thermo Fisher Scientific) and the direct-zol RNA MiniPrep kit (Zymo Research). The isolated RNA samples were then submitted to the Vanderbilt University Medical Center VANTAGE Core (Nashville, TN) for RNA quality determination and sequencing. Total RNA quality was assessed using a 2100 Bioanalyzer (Agilent, Santa Clara, CA). At least 200 ng of DNasetreated total RNA with high RNA integrity was used to generate poly-A-enriched mRNA libraries, using KAPA Stranded mRNA sample kits with indexed adaptors (New England BioLabs, Ipswich, MA). Library quality was assessed using the 2100 Bioanalyzer (Agilent), and libraries were quantitated using KAPA library Quantification kits (KAPA Biosystems). Pooled libraries were subjected to $150-\mathrm{bp}$ double-end sequencing using an Illumina NovaSeq6000 (San Diego, CA) according to the manufacturer's protocol. Bcl2fastq2 Conversion Software (Illumina) was used to generate de-multiplexed Fastq files.

\section{Analysis of RNA-Seq Data}

We used the RNA-seq data analysis tool Kallisto for read alignment and quantification (Bray et al., 2016). Kallisto pseudoaligns the reads to a reference, producing a list of transcripts that are compatible with each read while avoiding alignment of individual bases. In this study, we pseudo-aligned the reads to the Rattus norvegicus transcriptome (Rnor_6.0) downloaded

TABLE 1 | Viability of hepatocytes and renal proximal tubular epithelial cells exposed to thioacetamide-S-oxide compared to vehicle-exposed cells at 9 or $24 \mathrm{~h}$. The vehicle-exposed reference values for adenosine triphosphate (ATP) and lactate dehydrogenase $(\mathrm{LDH})$ are relative values at each time point.

\begin{tabular}{|c|c|c|c|c|c|}
\hline & & \multicolumn{2}{|c|}{$9 \mathrm{~h}$ exposure } & \multicolumn{2}{|c|}{$24 \mathrm{~h}$ exposure } \\
\hline & & ATP & LDH & ATP & LDH \\
\hline Type of cells & Dose (mM) & $\%$ & $\%$ & $\%$ & $\%$ \\
\hline \multirow[t]{3}{*}{ Hepatocytes } & 0 (vehicle) & $100 \pm 3$ & $100 \pm 4$ & $100 \pm 3$ & $100 \pm 3$ \\
\hline & 0.025 & $99 \pm 9$ & $103 \pm 6$ & $84 \pm 3$ & $98 \pm 7$ \\
\hline & 0.125 & $90 \pm 5$ & $99 \pm 5$ & $75 \pm 6$ & $95 \pm 5$ \\
\hline \multirow[t]{3}{*}{ Epithelial Cells } & 0 (vehicle) & $100 \pm 2$ & $100 \pm 9$ & $100 \pm 1$ & $100 \pm 8$ \\
\hline & 0.125 & $99 \pm 1$ & $103 \pm 11$ & $91 \pm 2$ & $99 \pm 6$ \\
\hline & 0.5 & $87 \pm 6$ & $95 \pm 11$ & $65 \pm 5$ & $90 \pm 9$ \\
\hline
\end{tabular}


from the Ensembl website (Zerbino et al., 2018). Kallisto achieves a level of accuracy similar to that of other competing methods, but is orders of magnitude faster than alternative methods. Its speed allows for the use of a bootstrapping technique to calculate uncertainties of transcript abundance estimates by repeating the analyses after resampling with replacement. In this study, we employed this technique to repeat the analysis 100 times. The files from RNA-seq analysis were deposited in NCBI's Gene Expression Omnibus (GEO) database under series accession numbers GSE120195 and GSE134569.

To identify differentially expressed genes (DEGs) from transcript abundance data, we used Kallisto's companion analysis tool Sleuth, which uses the results of the bootstrap analysis during transcript quantification to directly estimate the technical gene variance for each sample (Pimentel et al., 2017). DEGs were defined by using a false discovery rate adjusted $p$-value (q-value) of no more than 0.05 and a minimum gene expression $\beta$-value of 0.41 as the criteria for differential expression, which corresponds to a fold-change (FC) value of 1.5. Note that the $\beta$-value is defined as the natural logarithm of the effect size, and that the effect size and FC value of a gene are not equivalent. Nonetheless, the ranking and the directionality of change in gene expression (i.e., if a gene is up- or down-regulated) should be the same. In the Supplemental Material, we provide the q-values of all genes and the DEGs.

\section{KEGG Pathway Analysis}

To understand the biological significance of the alterations in gene expression levels induced by thioacetamide, we used the aggregated FC (AFC) method (Ackermann and Strimmer, 2009; Yu et al., 2017) to calculate significantly activated Kyoto Encyclopedia of Genes and Genomes (KEGG) pathways (Kanehisa and Goto, 2000). We downloaded the KEGG pathways from the Molecular Signatures Database (MsigDB) (c2.cp.kegg.v6.1.entrez.gmt) (Liberzon et al., 2011), provided by the Broad Institute, Cambridge, MA [http://software.broadinstitute.org/gsea/msigdb], in February 2018. This database contains pathway information curated from multiple online databases. Detailed descriptions and performance characteristics of the AFC method can be found in the original literature (Ackermann and Strimmer, 2009; Yu et al., 2017). In the AFC method, we first calculate the FC value for each gene (i.e., the difference between the mean log-transformed gene expression values for treatment and control conditions) and define the KEGG pathway score as the total FC value of all genes in the pathway. We then use the pathway scores to perform null hypothesis tests and estimate each pathway's significance by its p-value, defined as the probability that the pathway score for a random data set is greater than the score from the actual pathway. The sign of the pathway score represents the direction of regulation: the pathway is defined as up-regulated if the gene expression level after a treatment condition is increased relative to the control condition, and downregulated if it is instead reduced.

\section{Module Activation Score}

We developed the aggregate absolute FC (AAFC) method to calculate the activation score of a gene set (Schyman et al., 2018). This method identifies gene sets (e.g., modules) that are significantly changed. The AAFC method first calculates the FC value for each gene, i.e., the difference between the mean logtransformed gene expression values for samples in the treatment and control cohorts. We assessed the significance of this FC value by Student's t-test ( $\mathrm{n}=5$ for both treatment and control cohorts). We updated the procedure from that in our previous study (Schyman et al., 2018), in which we used "qualified" genes that passed Student's t-test ( $\mathrm{p}$-value $<0.05$ ). In the updated version, we included all genes, but calculated a combined p-value for each gene set (module) using Fisher's method as an indicator of robustness of the reliability of the genes in the module (Fisher, 1932). (See the Fisher values for all module calculations in Tables S2-S5 of the Supplemental Material.) The AAFC method then calculated the absolute value of each gene's log-transformed FC value, and for each gene set (e.g., module or pathway) calculated the total FC value of the absolute values. We then used the gene set scores to perform null hypothesis tests and estimated each gene set's significance by its p-value, defined as the probability that the score for randomly selected FC values (10,000 times) is greater than the score from the actual gene set. A small p-value implies that the gene set value is significant. The $\mathrm{z}$-score is the number of standard deviations by which the actual gene set value differs from the mean of the randomly selected FC values (10,000 times).

\section{Data Collection and Processing of High- and Low-Risk Liver Toxicants}

For the in vitro assessment of high- and low-risk liver toxicity, we used data from TG-GATEs (Igarashi et al., 2015), a publicly available database that contains data associating chemical exposures with transcriptomic changes in the liver of male Sprague-Dawley rats. We processed the data according to our previous protocol (Tawa et al., 2014), using the ArrayQualityMetrics-Bioconductor package (Kauffmann et al., 2008) to assess the quality of the Robust Multiarray Averaged (RMA) preprocessed data (Irizarry et al., 2003). In this process, we removed outlier arrays and renormalized the remaining data.

For the in vivo data, we used DrugMatrix, a publically available toxicogenomics database. This database contains a large collection of gene expression data obtained from SpragueDawley rats after exposure to a range of chemicals (Ganter et al., 2005). This dataset utilizes the Affymetrix GeneChip Rat Genome 230 2.0 Array. We used the same protocol as described in our previous publication (AbdulHameed et al., 2014).

\section{RESULTS}

\section{Differentially Expressed Genes (DEGs) Activated In Vitro and In Vivo}

Table 2 summarizes the number of DEGs identified in vivo from liver and kidney exposed to thioacetamide treatment for 8 or $24 \mathrm{~h}$ and in vitro from primary hepatocytes and renal proximal tubular epithelial cells exposed to the thioacetamide metabolite, thiacetamide-S-oxide, for 9 or $24 \mathrm{~h}$. The two time points and two doses of thioacetamide-S-oxide (see Table 1) produced minimal cytotoxicity, as indicated by two different cell viability assays. Thus, we attempted to mimic the level of toxicity induced 
TABLE 2 | Differentially expressed genes after exposure in vivo to thioacetamide and in vitro to thioacetamide-S-oxide.

\begin{tabular}{|c|c|c|c|c|c|c|c|c|}
\hline & \multicolumn{4}{|c|}{ In vivo } & \multicolumn{4}{|c|}{ In vitro } \\
\hline & \multicolumn{2}{|c|}{ Low dose } & \multicolumn{2}{|c|}{ High dose } & \multicolumn{2}{|c|}{ Low dose } & \multicolumn{2}{|c|}{ High dose } \\
\hline & $8 \mathrm{~h}$ & $24 \mathrm{~h}$ & $8 \mathrm{~h}$ & $24 \mathrm{~h}$ & $9 \mathrm{~h}$ & $24 \mathrm{~h}$ & $9 \mathrm{~h}$ & $24 \mathrm{~h}$ \\
\hline Liver & 3027 & 1999 & 4443 & 4307 & 259 & 4292 & 2159 & 3178 \\
\hline Kidney & 257 & 746 & 172 & 1571 & 890 & 71 & 2575 & 3529 \\
\hline
\end{tabular}

by thioacetamide and its metabolites in vivo without attempting to match toxicant levels or time points exactly with those used previously for in vitro studies. The goal was to identify genes whose expression changes in vivo are associated with early injury induced by thioacetamide and/or its metabolites, which may also be altered similarly in rat primary liver and kidney cells following in vitro exposure to thioacetamide-S-oxide. In most cases, the number of DEGs identified in the liver and kidney depended on the dose (Table 2) with two exceptions: in vitro hepatocytes showed 4,292 DEGs $24 \mathrm{~h}$ after low-dose treatment compared to 3,178 DEGs $24 \mathrm{~h}$ after high-dose treatment, and in vivo kidneys showed 257 DEGs $8 \mathrm{~h}$ after low-dose treatment compared to 172 DEGs $8 \mathrm{~h}$ after high-dose treatment. Another notable trend in the in vivo study was that all kidney samples showed fewer DEGs than liver samples, consistent with the notion that the liver is responsible for metabolizing the majority of thioacetamide to reactive metabolites like thioacetamide-S-oxide. Thus, the liver was likely exposed to higher concentrations of this toxic thioacetamide metabolite, even though the in vivo dose of thioacetamide administered to the rats was the same for the liver and kidney. In contrast, rat liver and kidney primary cells were exposed in vitro directly to a high- and low-dose of the thioacetamide metabolite, thioacetamide-S-oxide. A high and low dose of thioacetamide-S-oxide was selected for each cell type to provide a similar level of cytotoxicity between each cell type (see Table 1). This led to a higher in vitro dose for renal tube epithelial cells than for hepatocytes, which might explain the liver and kidney discrepancy between the in vivo and in vitro results.

Figure 1 shows the number of DEGs overlapping between those observed in vivo and in vitro for the liver and kidney 24 $\mathrm{h}$ after high-dose exposure to thioacetamide/thioacetamide-Soxide. The overlap as a proportion of the total number of DEGs

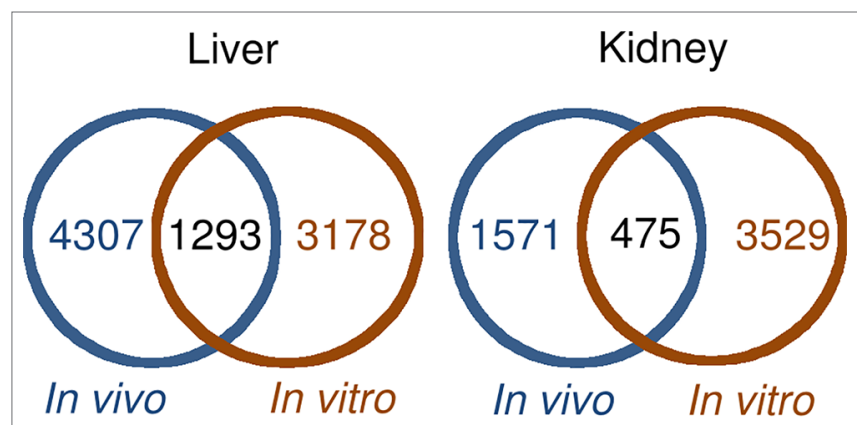

Thioacetamide High dose 24 hours

FIGURE 1 | Overlap between differentially expressed genes in in vivo and in vitro $24 \mathrm{~h}$ after thioacetamide/thioacetamide-S-oxide exposure. is small for the liver and kidney, covering approximately $20 \%$ and $10 \%$, respectively. The overlapping genes might still be useful as predictors if they were correlated. However, an analysis of the relative gene expression changes in vivo and in vitro revealed no correlation for either the liver or kidney (Figure 2).

\section{KEGG Pathway Activation In Vitro and In Vivo}

For our analysis, we used the KEGG pathway database (Kanehisa and Goto, 2000). To identify activated pathways we used the AFC method (Ackermann and Strimmer, 2009), which performs well compared to other popular pathway analysis methods (Yu et al., 2017). The AFC procedure uses all genes in a pathway to calculate the FC value and determine the significance by calculating the p-value using randomly selected genes (see Materials and Methods section).

Table 3 summarizes the KEGG pathways that were significantly activated, with a $\mathrm{p}$-value of $<0.05$, both in vivo and in vitro for the liver and kidney $24 \mathrm{~h}$ after high-dose exposure to a form of thioacetamide. The in vivo and in vitro results showed overlap for 12 pathways in the liver and 13 pathways in the kidney. Changing the significance threshold to $\mathrm{p}<0.01$ resulted in only two overlapping pathways (cardiac muscle contraction and oxidative phosphorylation), which appeared both in the liver and the kidney. However, there were no overlapping pathways in the liver when we considered the directionality, whether a pathway was significantly overexpressed or suppressed (up- or down-regulated). Table S1 in the Supplemental Material shows the KEGG pathways with their calculated p-values, $24 \mathrm{~h}$ after high-dose treatment in vivo and in vitro for both the liver and kidney.

\section{Injury Module Activation Analysis}

We previously identified and evaluated 8 kidney and 11 liver injury modules (Te et al., 2016; Schyman et al., 2018). In those studies, we calculated the average absolute $\log _{2} \mathrm{FC}$ value of all genes in a module to determine the activation score for each injury module. Here, we modified the procedure from our previous study (Schyman et al., 2018), where we only used genes that passed the t-test criteria, and instead included all genes. Tables 4-7 show, in bold, the z-score values of significantly activated injury modules, for which the $\mathrm{p}$-value was less than 0.01. Tables S2-S5 in the Supplemental Material include the calculated p-values. For practical purposes, one should identify the top-ranking modules with the highest $\mathrm{z}$-score values as the most likely injury phenotypes. However, several injury phenotypes may coexist, such as necrosis and cellular infiltration, which are early inflammatory responses.

\section{Liver Module Activation In Vivo}

In rats, thioacetamide exposure significantly activated ( $\mathrm{p}$-value $<0.01$ ) several injury modules in the liver (Table 4). For each condition, the injury modules were ranked by the $\mathrm{z}$-score from the most to the least likely injury endpoint. $8 \mathrm{~h}$ after thioacetamide exposure, all liver injury modules significantly activated at the low dose were also activated at the high dose, albeit with different ranking orders. Similarly, $24 \mathrm{~h}$ after lowdose exposure, all significantly activated injury modules were 

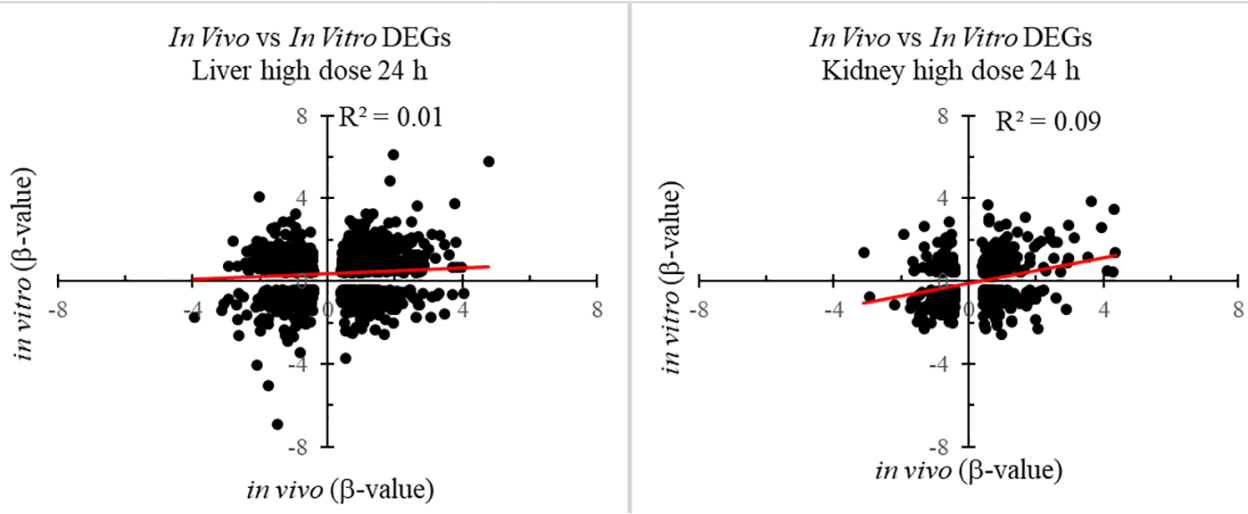

FIGURE 2 | Correlation between differentially expressed genes (DEGs) in in vitro and in vivo $24 \mathrm{~h}$ after thioacetamide/thioacetamide-S-oxide exposure.

TABLE 3 | Overlap of Kyoto Encyclopedia of Genes and Genomes (KEGG) pathways significantly activated ( $\mathrm{p}$-value $<0.05$ ) in vivo and in vitro, $24 \mathrm{~h}$ after high-dose thioacetamide/thioacetamide-S-oxide exposure.

\begin{tabular}{|c|c|c|c|}
\hline \multirow[t]{2}{*}{$\begin{array}{l}\text { KEGG pathway } \\
\text { ( } p \text {-value }<0.05 \text {; bold } p \text {-value }<0.01 \text { ) }\end{array}$} & \multicolumn{2}{|c|}{$\begin{array}{l}\text { In vivo-In vitro } \\
\text { overlap }\end{array}$} & \multirow[t]{2}{*}{ Function } \\
\hline & Liver & Kidney & \\
\hline Limonene and pinene degradation & $\sqrt{ }$ & & Metabolism \\
\hline PPAR signaling pathway & $\sqrt{ }$ & & Endocrine system \\
\hline Ascorbate and aldarate metabolism & $\sqrt{ }$ & & Metabolism \\
\hline Butanoate metabolism & $\sqrt{ }$ & & Metabolism \\
\hline Tyrosine metabolism & $\sqrt{ }$ & & Metabolism \\
\hline Cardiac muscle contraction ${ }^{a}$ & $\sqrt{ }$ & $\sqrt{ }$ & $\begin{array}{l}\text { Circulatory } \\
\text { system }\end{array}$ \\
\hline Antigen processing and presentation & $\sqrt{ }$ & $\sqrt{ }$ & Immune system \\
\hline Oxidative phosphorylation & $\sqrt{ }$ & $\sqrt{ }$ & Metabolism \\
\hline Glutathione metabolism & $\sqrt{ }$ & $\sqrt{ }$ & Metabolism \\
\hline Linoleic acid metabolism & $\sqrt{ }$ & $\sqrt{ }$ & Metabolism \\
\hline $\begin{array}{l}\text { Metabolism of xenobiotics by cytochrome } \\
\text { P450 }\end{array}$ & $\sqrt{ }$ & $\sqrt{ }$ & Metabolism \\
\hline Drug metabolism cytochrome P450 & $\sqrt{ }$ & $\sqrt{ }$ & Metabolism \\
\hline Pentose and glucuronate interconversions & & $\sqrt{ }$ & Metabolism \\
\hline Arginine and proline metabolism & & $\sqrt{ }$ & Metabolism \\
\hline Taurine and hypotaurine metabolism & & $\sqrt{ }$ & Metabolism \\
\hline Selenoamino acid metabolism & & $\sqrt{ }$ & Metabolism \\
\hline Porphyrin and chlorophyll metabolism & & $\sqrt{ }$ & Metabolism \\
\hline Nitrogen metabolism & & $\sqrt{ }$ & Metabolism \\
\hline
\end{tabular}

${ }^{a}$ Bold text indicates significantly activated pathway with a p-value $<0.01$.

also activated after high-dose exposure. There was a significant overlap between the top-ranked liver injury modules at $24 \mathrm{~h}$ after low- and high-dose exposures. The difference in z-scores of the injury modules between 8 and $24 \mathrm{~h}$ were more pronounced than between low- and high-dose treatments.

\section{Kidney Module Activation In Vivo}

Thioacetamide exposures significantly activated ( $\mathrm{p}$-value $<0.01$ ) only a few injury modules in the kidney (Table 5). Necrosis was consistently the top-ranked kidney injury module in all kidney samples after thioacetamide exposure. At $24 \mathrm{~h}$ after high-dose exposure, several kidney injury modules were significantly activated, but necrosis was still the top-ranked injury phenotype.

\section{Liver Module Activation In Vitro}

Table 6 shows the liver injury modules activated by thioacetamide$\mathrm{S}$-oxide (the reactive metabolite of thioacetamide) in hepatocytes. $9 \mathrm{~h}$ after low- and high-dose treatments, no liver injury modules were significantly activated ( $\mathrm{p}$-value $<0.01$ ). However, after $24 \mathrm{~h}$, fibrosis was among the two highest-ranked injury modules for both low- and high-dose treatments. All five injury modules that were significantly activated $24 \mathrm{~h}$ after the low-dose treatment were also significantly activated after the high-dose treatment.

\section{Kidney Module Activation In Vitro}

Table 7 shows the kidney injury module activation scores for thioacetamide-S-oxide in renal proximal tubular epithelial cells. None of the high-dose treatments significantly activated any injury modules, but $9 \mathrm{~h}$ after exposure to the low-dose treatment the kidney injury module, fibrosis, was slightly activated. However, this injury module was not significantly (p-value < 0.01 ) activated at $24 \mathrm{~h}$ after treatment.

\section{In Vitro-In Vivo Correlation}

At $24 \mathrm{~h}$ after the high-dose treatment, three of the four liver injury modules that were ranked highest in vitro could be found among the four highest-ranked modules in vivo (Tables 4 and 6). Fibrosis was the second-highest ranked injury module in vitro and in vivo. These results are in agreement with fibrosis being the primary injury endpoint of thioacetamide exposure.

Figure 3 shows the correlation between in vivo and in vitro experiments for different liver and kidney samples. The injury modules activated in the liver $24 \mathrm{~h}$ after high- and low-dose treatment were strongly correlated $\left(\mathrm{R}^{2}>0.6\right)$. However, those activated in the kidney $24 \mathrm{~h}$ after high-dose and low-dose treatment showed no correlation. There was no positive in vivo-in vitro correlation at the shorter time points, regardless of the dose.

\section{In Vitro Predictions of High- and Low-Risk Liver Toxicants}

In this section, we extend our validation to identify key injury phenotypes and to differentiate high-risk liver toxicants from low-risk toxicants. To this end, we identified four compounds 
TABLE 4 | Activation of liver injury modules in vivo from rat liver tissue after exposure to thioacetamide.

\begin{tabular}{|c|c|c|c|c|c|c|c|}
\hline \multicolumn{4}{|c|}{ Low dose } & \multicolumn{4}{|c|}{ High dose } \\
\hline \multicolumn{2}{|l|}{$8 \mathrm{~h}$} & \multicolumn{2}{|c|}{$24 \mathrm{~h}$} & \multicolumn{2}{|l|}{$8 \mathrm{~h}$} & \multicolumn{2}{|l|}{$24 \mathrm{~h}$} \\
\hline Module & z-score & Module & z-score & Module & z-score & Module & z-score \\
\hline $\begin{array}{l}\text { Oval cell } \\
\text { proliferationa }^{a}\end{array}$ & 8.5 & Anisonucleosis & 14.7 & Cellular infiltration & 6.8 & Cellular infiltration & 14.9 \\
\hline Single cell necrosis & 6.7 & Cellular infiltration & 14.2 & Single cell necrosis & 6.0 & Fibrosis & 12.0 \\
\hline Nuclear alteration & 5.6 & Fibrosis & 11.1 & Oval cell proliferation & 4.6 & Cellular foci & 10.0 \\
\hline Cellular infiltration & 5.2 & Oval cell proliferation & 9.6 & Cellular foci & 3.8 & Anisonucleosis & 9.1 \\
\hline Anisonucleosis & 3.4 & Cellular foci & 9.0 & Anisonucleosis & 3.7 & Oval cell proliferation & 8.0 \\
\hline Bile duct proliferation & 3.2 & Single cell necrosis & 4.6 & Fibrosis & 3.1 & Single cell necrosis & 6.5 \\
\hline Cellular foci & 1.8 & Nuclear alteration & 4.1 & Bile duct proliferation & 3.1 & Nuclear alteration & 5.0 \\
\hline Hematopoiesis & 1.5 & Bile duct proliferation & 3.6 & Nuclear alteration & 2.4 & Bile duct proliferation & 4.8 \\
\hline Fibrosis & 1.1 & Hematopoiesis & 2.9 & Hematopoiesis & 2.3 & Hematopoiesis & 3.4 \\
\hline Cytoplasmic alteration & 0.8 & Granular degeneration & 1.4 & Cytoplasmic alteration & 1.1 & Cytoplasmic alteration & -0.5 \\
\hline Granular degeneration & -0.8 & Cytoplasmic alteration & 0.2 & Granular degeneration & 0.2 & Granular degeneration & -0.9 \\
\hline
\end{tabular}

${ }^{a}$ Bold text indicates significantly activated module ( $p$-value $\left.<0.01\right)$.

TABLE 5 | Activation of kidney injury modules in vivo from rat kidney tissue after exposure to thioacetamide.

\begin{tabular}{|c|c|c|c|c|c|c|c|}
\hline \multicolumn{4}{|c|}{ Low dose } & \multicolumn{4}{|c|}{ High dose } \\
\hline \multicolumn{2}{|c|}{$8 \mathrm{~h}$} & \multicolumn{2}{|l|}{$24 \mathrm{~h}$} & \multicolumn{2}{|l|}{$8 \mathrm{~h}$} & \multicolumn{2}{|l|}{$24 \mathrm{~h}$} \\
\hline Module & z-score & Module & z-score & Module & z-score & Module & z-score \\
\hline Necrosis $^{a}$ & 5.2 & Necrosis & 2.6 & Necrosis & 7.3 & Necrosis & 15.3 \\
\hline Dilatation & 0.6 & Dilatation & 1.6 & Cellular infiltration & 1.7 & Cellular infiltration & 11.2 \\
\hline Degeneration & 0.2 & Degeneration & 0.9 & Degeneration & 1.5 & Degeneration & 11.2 \\
\hline Cellular infiltration & -1.8 & Cellular infiltration & 0.7 & Hyaline cast & 0.6 & Hyaline cast & 6.4 \\
\hline Hyaline cast & -2.0 & Hyaline cast & -0.5 & Hypertrophy & 0.2 & Dilatation & 6.4 \\
\hline Hypertrophy & -2.1 & Hypertrophy & -1.0 & Intracytoplasmic inclusion body & -0.3 & Fibrosis & 1.5 \\
\hline $\begin{array}{l}\text { Intracytoplasmic } \\
\text { inclusion body }\end{array}$ & -2.4 & $\begin{array}{l}\text { Intracytoplasmic inclusion } \\
\text { body }\end{array}$ & -2.7 & Dilatation & -0.7 & $\begin{array}{l}\text { Intracytoplasmic inclusion } \\
\text { body }\end{array}$ & -0.1 \\
\hline Fibrosis & -3.7 & Fibrosis & -3.0 & Fibrosis & -0.9 & Hypertrophy & -1.7 \\
\hline
\end{tabular}

abold text indicates significantly activated module ( $p$-value $<0.01)$.

TABLE 6 | Activation of liver injury modules in vitro from rat hepatocytes after exposure to the thioacetamide metabolite, thioacetamide-S-oxide.

Low dose

\begin{tabular}{|c|c|c|c|}
\hline \multicolumn{2}{|l|}{$9 \mathrm{~h}$} & \multicolumn{2}{|c|}{$24 \mathrm{~h}$} \\
\hline Module & z-score & Module & z-score \\
\hline Hematopoiesis & -0.4 & Fibrosis $^{a}$ & 6.4 \\
\hline Anisonucleosis & -1.3 & Anisonucleosis & 5.6 \\
\hline Granular degeneration & -1.5 & Cellular foci & 5.2 \\
\hline Fibrosis & -1.8 & Bile duct proliferation & 3.4 \\
\hline Bile duct proliferation & -1.8 & Cellular infiltration & 3.3 \\
\hline Cytoplasmic alteration & -2.2 & Single cell necrosis & 2.4 \\
\hline Cellular infiltration & -2.2 & Oval cell proliferation & 2.2 \\
\hline Cellular foci & -2.3 & Nuclear alteration & 0.2 \\
\hline Single cell necrosis & -2.4 & Hematopoiesis & 0.0 \\
\hline Oval cell proliferation & -3.8 & Granular degeneration & -1.6 \\
\hline Nuclear alteration & -4.5 & Cytoplasmic alteration & -1.6 \\
\hline
\end{tabular}

${ }^{a}$ Bold text indicates significantly activated module ( $p$-value $\left.<0.01\right)$.

with in vitro data from TG-GATEs and corresponding in vivo data from DrugMatrix. We selected carbon tetrachloride and lomustine as high-risk toxicants, which are known to promote fibrosis in rats after 29 days of exposure to $300 \mathrm{mg} / \mathrm{kg}$ of carbon
High dose

\begin{tabular}{|c|c|c|c|}
\hline \multicolumn{2}{|c|}{$9 \mathrm{~h}$} & \multicolumn{2}{|l|}{$24 \mathrm{~h}$} \\
\hline Module & z-score & Module & z-score \\
\hline Hematopoiesis & 1.0 & Anisonucleosis & 6.9 \\
\hline Anisonucleosis & 0.3 & Fibrosis & 6.5 \\
\hline Single cell necrosis & 0.1 & Cellular foci & 6.1 \\
\hline Granular degeneration & -0.7 & Bile duct proliferation & 3.7 \\
\hline Fibrosis & -0.9 & Cellular infiltration & 3.6 \\
\hline Vuclear alteration & -0.9 & Oval cell proliferation & 1.2 \\
\hline Cellular foci & -1.1 & Single cell necrosis & 1.0 \\
\hline Oval cell proliferation & -1.4 & Nuclear alteration & 0.1 \\
\hline Bile duct proliferation & -2.1 & Hematopoiesis & -0.2 \\
\hline Cellular infiltration & -2.1 & Cytoplasmic alteration & -1.6 \\
\hline Cytoplasmic alteration & -2.2 & Granular degeneration & -1.9 \\
\hline
\end{tabular}

tetrachloride and after 29 days of exposure to $6 \mathrm{mg} / \mathrm{kg}$ of lomustine (Igarashi et al., 2015). The doses used in DrugMatrix were similar: $400 \mathrm{mg} / \mathrm{kg}$ for carbon tetrachloride and 4.20 and $8.75 \mathrm{mg} / \mathrm{kg}$ for lomustine (Ganter et al., 2005). We classified 
TABLE 7 | Activation of kidney injury modules in vitro from renal tube epithelial cells after exposure to thioacetamide metabolite, thiacetamide-S-oxide.

\begin{tabular}{|c|c|c|c|c|c|c|c|}
\hline \multicolumn{4}{|c|}{ Low dose } & \multicolumn{4}{|c|}{ High dose } \\
\hline \multicolumn{2}{|l|}{$9 \mathrm{~h}$} & \multicolumn{2}{|l|}{$24 \mathrm{~h}$} & \multicolumn{2}{|l|}{$9 \mathrm{~h}$} & \multicolumn{2}{|l|}{$24 \mathrm{~h}$} \\
\hline Module & z-score & Module & z-score & Module & z-score & Module & z-score \\
\hline Fibrosis ${ }^{a}$ & 3.3 & Hypertrophy & 1.6 & Hypertrophy & 1.8 & Cellular infiltration & 1.9 \\
\hline Hypertrophy & 1.7 & Intracytoplasmic inclusion body & -0.2 & Necrosis & 1.3 & Fibrosis & 1.1 \\
\hline Cellular infiltration & 1.3 & Fibrosis & -0.3 & Degeneration & 1.2 & Intracytoplasmic inclusion body & 1.1 \\
\hline Degeneration & 1.0 & Cellular infiltration & -1.1 & Dilatation & 1.1 & Hypertrophy & 1.0 \\
\hline Dilatation & 0.8 & Dilatation & -1.1 & Fibrosis & 1.1 & Necrosis & 0.5 \\
\hline Intracytoplasmic inclusion body & 0.0 & Necrosis & -1.2 & Cellular infiltration & 0.4 & Dilatation & 0.1 \\
\hline Hyaline cast & -0.2 & Hyaline cast & -1.3 & Intracytoplasmic inclusion body & 0.2 & Hyaline cast & -0.5 \\
\hline Necrosis & -0.6 & Degeneration & -1.8 & Hyaline cast & -0.6 & Degeneration & -1.2 \\
\hline
\end{tabular}

abold text indicates significantly activated module ( $p$-value $<0.01)$.

naproxen and tamoxifen as low-risk toxicants, based on the LiverTox website (Hoofnagle et al., 2013), as liver fibrosis was not detected in the histology reports from TG-GATEs.

The in vitro data from TG-GATEs contain repeated-dose exposure to chemicals at low, medium, and high doses at three different time-points $(2,8$, and $24 \mathrm{~h})$. For this analysis, we calculated a module activation score for each condition and selected the maximum score. We provide all activation scores in Table S6 (Supplemental Material).

Table 8 shows the maximum injury module activation scores from in vitro and in vivo expression data for the four compounds. The fibrosis module was significantly activated for the two highrisk compounds in both in vitro and in vivo, but not for the lowrisk compounds.

\section{DISCUSSION}

We used different approaches to assess the correlation between in vitro and in vivo toxicogenomic experiments. No positive correlations were observed for liver or kidney injury modules 8 or $9 \mathrm{~h}$ after thioacetamide/thioacetamide-S-oxide exposure. However, our injury module approach did indicate a strong correlation $\left(\mathrm{R}^{2}>0.60\right)$ at $24 \mathrm{~h}$ after high-dose treatment of thioacetamide/thioacetamide-S-oxide between liver injury modules activated in vitro (in hepatocytes) and those activated in vivo (in rats), but no correlation $\left(\mathrm{R}^{2}<0.10\right)$ between kidney injury modules activated in vitro (in renal cells) and those activated in vivo (in rats). Interestingly, there was also a strong in vitro-in vivo correlation $\left(\mathrm{R}^{2}=0.66\right)$ for liver injury modules activated $24 \mathrm{~h}$ after low-dose treatment, which further indicates the sensitivity of the injury module approach to identifying specific injury phenotypes even after a low-dose exposure.

A noteworthy observation for the activation of liver injury modules in vivo and in vitro was the increase in activation score and rank of the fibrosis module over time ( 8 to $24 \mathrm{~h}$ ) for both low- and high-dose treatments (see Tables 4 and 6). The opposite trend was observed for the single cell necrosis module in vivo, which was the top-ranked module $8 \mathrm{~h}$ after exposure but a midranked module after $24 \mathrm{~h}$ (see Table 4 ). These observations are reasonable, given that fibrosis requires more time to develop than single cell necrosis with regard to an early immune response.
DEGs in vitro showed little overlap with DEGs in vivo (Figure 1), and gene FC values among the overlapping genes were uncorrelated (Figure 2). These data suggest that any in vitro-in vivo correlation based solely on individual genes are likely to be coincidental.

The KEGG pathway analysis of in vivo gene expression data from rats exposed to thioacetamide identified multiple biologically relevant pathways in the liver, as discussed previously (Schyman et al., 2018). In the in vitro hepatocyte experiment, 17 pathways (excluding human disease pathways) were significantly activated with a p-value of less than 0.05 , which included 13 metabolic pathways, as well as cardiac muscle contraction, antigen processing and isoleucine biosynthesis, PPAR signaling, and complement and coagulation cascades pathways (see Supplemental Material for all activated KEGG pathways). Among the 17 pathways activated in vitro, 13 overlapped with the pathways activated in liver (in vivo) (Table 3). Nine of these pathways were related to metabolism (e.g., xenobiotic biodegradation and metabolism, carbohydrate metabolism, amino acid metabolism) and three others were related to the circulatory, immune, and endocrine systems (i.e., cardiac muscle contraction, antigen processing and isoleucine biosynthesis, and PPAR signaling pathways). Similarly, the majority of the significantly activated KEGG pathways in the kidney that showed in vitro-in vivo overlap were involved in metabolism (Table 3), and also circulatory and immune systems (i.e., cardiac muscle contraction and antigen processing and isoleucine biosynthesis). Table 3 shows that more than half of the in vitro-in vivo overlapped pathways in liver or kidney also overlapped between liver and kidney samples. Some of these pathways were expected, such as metabolism of xenobiotics by cytochrome $\mathrm{P} 450$ and drug metabolism cytochrome p450, but also cardiac muscle contraction, antigen processing and isoleucine biosynthesis were common across organs. It is interesting to note that the glutathione metabolism pathway was also common, which is important in antioxidant defense and cellular function (e.g., cell proliferation, apoptosis, immune response) (Wu et al., 2004). However, these pathways, which are involved in general biological responses and are not injury specific, showed low specificity between the liver and kidney.

Previous in vitro-in vivo research has often focused on identifying correlated genes, pathways, or gene ontology terms (Zhang et al., 2013; De Abrew et al., 2015; Sutherland et al., 2016; 
In Vivo vs In Vitro

Liver Injury Module Activation

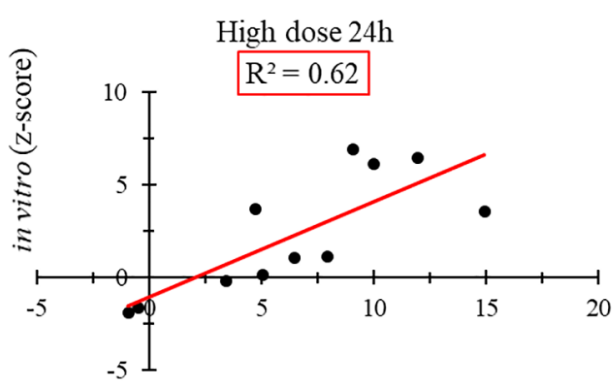

High dose $8 \mathrm{~h}$

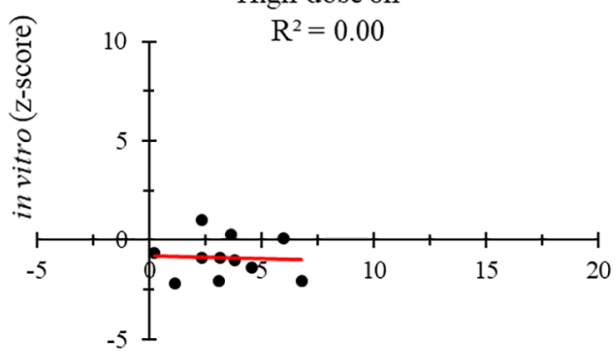

Low dose $24 \mathrm{~h}$

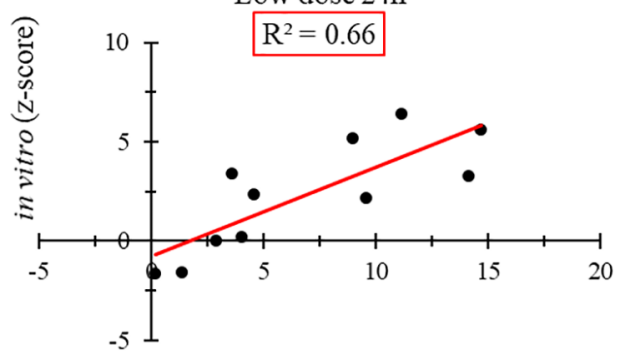

Low dose $8 \mathrm{~h}$

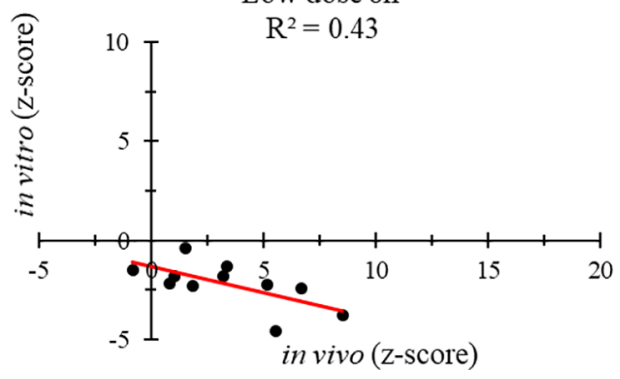

In Vivo vs In Vitro

Kidney Injury Module Activation

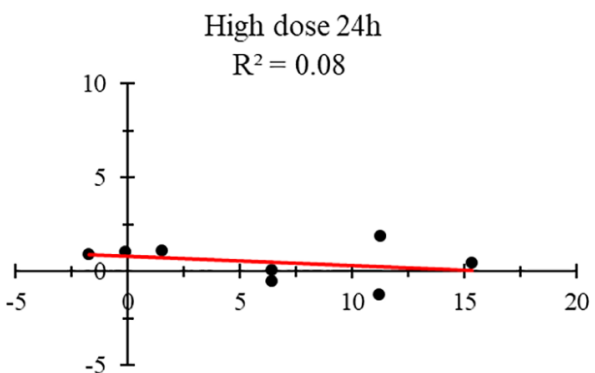

High dose $8 \mathrm{~h}$

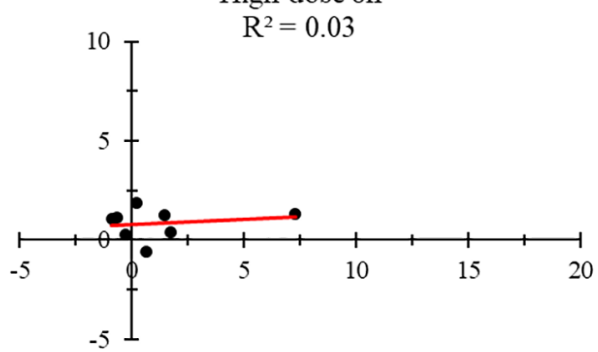

Low dose $24 \mathrm{~h}$
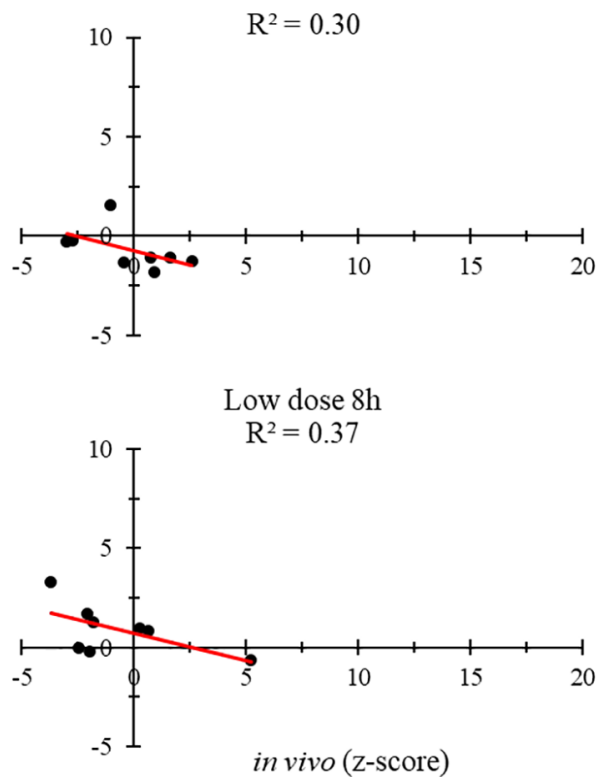

FIGURE 3 | Correlation between injury modules activated in vivo and in vitro.

Van den Hof et al., 2017; Taškova et al., 2018). We have shown that DEGs do not show satisfactory in vitro-in vivo correlations, which makes selection of individual genes indicative of injury unreliable. Furthermore, our KEGG pathway analysis identified several overlapping pathways between in vivo and in vitro conditions, but no clear link to fibrosis, the liver injury most associated with thioacetamide exposure.
Here, we show that our modular approach to identify gene sets specific to injury phenotypes performed well in assessing in vivo results from in vitro gene expression data after exposure to a thioacetamide metabolite, thioacetamide-S-oxide. An important factor in our modular approach is the use of absolute FC values to identify activated injury modules. Although this has been shown to be important in pathway analysis (Ackermann and Strimmer, 
TABLE 8 | Activation of liver injury modules in vitro and in vivo in rat hepatocytes after exposure to high- and low-risk liver toxicants. Bold text indicates significant activation of the Fibrosis module ( $p$-value $<0.01)$.

High-risk liver toxicants

Carbon tetrachloride

\begin{tabular}{|c|c|c|c|c|c|c|c|}
\hline \multicolumn{2}{|c|}{ In vivo (DrugMatrix) } & \multicolumn{2}{|c|}{ In vivo (TG-GATEs) ${ }^{b}$} & \multicolumn{2}{|c|}{ In vivo (DrugMatrix) ${ }^{c}$} & \multicolumn{2}{|c|}{ In vitro (TG-GATEs) ${ }^{d}$} \\
\hline Anisonucleosis & 12.0 & Fibrosis & 4.5 & Fibrosis & 10.2 & Fibrosis & 2.9 \\
\hline Fibrosis & 11.1 & Anisonucleosis & 4.4 & Cellular infiltration & 8.3 & Anisonucleosis & 2.4 \\
\hline Cellular infiltration & 10.5 & Hematopoiesis & 3.7 & Cellular foci & 7.7 & Cellular infiltration & 2.2 \\
\hline Cellular foci & 8.2 & Cellular foci & 2.8 & Single cell necrosis & 4.8 & Granular degeneration & 2.0 \\
\hline Single cell necrosis & 5.9 & Cellular infiltration & 1.8 & Hematopoiesis & 4.5 & Oval cell proliferation & 1.9 \\
\hline Nuclear alteration & 3.4 & Nuclear alteration & 1.7 & Anisonucleosis & 2.4 & Bile duct proliferation & 1.9 \\
\hline Hematopoiesis & 2.2 & Granular degeneration & 1.7 & Bile duct proliferation & 2.2 & Hematopoiesis & 1.7 \\
\hline Oval cell proliferation & 1.6 & Single cell necrosis & 1.3 & Oval cell proliferation & 2.0 & Cellular foci & 1.6 \\
\hline Granular degeneration & 0.3 & Cytoplasmic alteration & 1.0 & Cytoplasmic alteration & 1.3 & Cytoplasmic alteration & 0.7 \\
\hline Cytoplasmic alteration & -0.1 & Oval cell proliferation & 0.8 & Granular degeneration & 0.9 & Nuclear alteration & 0.1 \\
\hline Bile duct proliferation & -0.3 & Bile duct proliferation & 0.7 & Nuclear alteration & 0.4 & Single cell necrosis & -0.2 \\
\hline
\end{tabular}

Low-risk liver toxicants

\begin{tabular}{|c|c|c|c|c|c|c|c|}
\hline \multicolumn{4}{|c|}{ Naproxen } & \multicolumn{4}{|c|}{ Tamoxifen } \\
\hline \multicolumn{2}{|c|}{ In vivo (DrugMatrix)e } & \multicolumn{2}{|c|}{ In vivo (TG-GATEs) ${ }^{f}$} & \multicolumn{2}{|c|}{ In vivo (DrugMatrix) ${ }^{g}$} & \multicolumn{2}{|c|}{ In vitro (TG-GATEs) ${ }^{\mathrm{h}}$} \\
\hline Nuclear alteration & 0.3 & Granular degeneration & 4.5 & Anisonucleosis & 3.4 & Anisonucleosis & 4.6 \\
\hline Cytoplasmic alteration & 0.0 & Hematopoiesis & 2.4 & Granular degeneration & 1.8 & Hematopoiesis & 2.7 \\
\hline Single cell necrosis & -0.3 & Cytoplasmic alteration & 1.9 & Cytoplasmic alteration & 1.1 & Fibrosis & 1.8 \\
\hline Oval cell proliferation & -0.5 & Nuclear alteration & 1.9 & Nuclear alteration & 0.2 & Cytoplasmic alteration & 1.7 \\
\hline Bile duct proliferation & -1.7 & Bile duct proliferation & 1.7 & Single cell necrosis & 0.1 & Granular degeneration & 1.6 \\
\hline Anisonucleosis & -2.1 & Anisonucleosis & 1.5 & Fibrosis & -0.4 & Oval cell proliferation & 1.4 \\
\hline Cellular foci & -2.4 & Oval cell proliferation & 1.2 & Cellular foci & -0.5 & Nuclear alteration & 1.4 \\
\hline Cellular infiltration & -2.6 & Fibrosis & 1.1 & Cellular infiltration & -0.7 & Cellular infiltration & 1.0 \\
\hline Granular degeneration & -2.6 & Single cell necrosis & 0.9 & Bile duct proliferation & -0.9 & Cellular foci & 0.8 \\
\hline Fibrosis & -2.9 & Cellular foci & 0.8 & Hematopoiesis & -1.0 & Single cell necrosis & 0.1 \\
\hline Hematopoiesis & -3.9 & Cellular infiltration & 0.1 & Oval cell proliferation & -1.0 & Bile duct proliferation & -0.1 \\
\hline
\end{tabular}

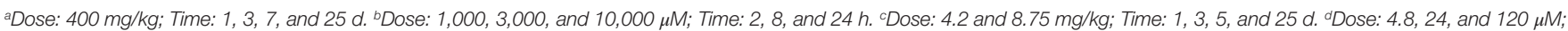

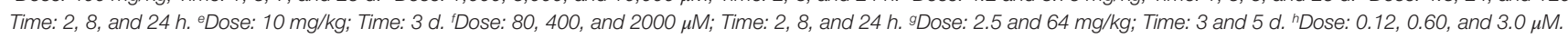

2009), it leads to loss of information about the directionality of change in the activated pathway. However, our results showed weak in vivo-in vitro correlations for the directionality of the FC (Figure 2), suggesting that the use of absolute FC values is critical to the success of the injury module approach.

Previous studies have typically focused on identifying gene signatures for a single injury phenotype (e.g., cancer, steatosis, cholestasis) (Segal et al., 2004; Sahini et al., 2014; Parmentier et al., 2017). Although this approach is adequate for understanding the underlying biological mechanisms of toxicity, a key aspect of predicting a specific organ injury phenotype is the ability to test and rank different injury phenotypes to identify the most likely injury. The prediction should also be organ-specific, which requires different sets of injury modules for different organs. Other studies have used similar multi-injury-gene set approaches to focus on liver injuries (De Abrew et al., 2015; Sutherland et al., 2017; Sutherland et al., 2019) or kidney injuries (Minowa et al., 2012).

A major limitation of our injury module approach is that we can only predict injuries for the injury modules we have identified. There are many more injury phenotypes for which we have not yet been able to assign a gene set, because publicly available data are still limited. Other limitations of our approach are that injury phenotypes are not necessarily indistinguishable, and that some injuries respond at different time scales (e.g., cellular infiltration is often an early response, whereas fibrosis is more pronounced later when the injury is more advanced). Additionally, our approach does not yet consider injury modules for other organs, such as the heart and brain.

To further validate the ability of our injury modules to predict in vivo injury endpoints, we selected four compounds with in vitro data from TG-GATEs and corresponding in vivo data in DrugMatrix. We did not expect perfect in vitro-in vivo concordance because the doses and time points were not necessarily determined in the same way (i.e., the highest dose with the least toxic response). However, our aim was to test whether we could identify a key injury phenotype and differentiate high-risk liver toxicants known to cause fibrosis from low-risk toxicants. Figure 4 shows the significantly activated injury modules based on the activation scores in Table 8. For easy comparison we highlighted the fibrosis module in pink and the radius indicate the significance. We found that the injury module approach differentiated toxicants from non-toxicants based on activation scores and identified fibrosis as one of the injury phenotypes among the high-risk toxicants. Nontoxicants, when presented alone, only activated a few benign injury modules significantly.

In summary, our results support the hypothesis that coexpressed gene sets specific to an injury phenotype (injury modules) may be useful to predict in vivo injury endpoints, using RNA-seq data from 


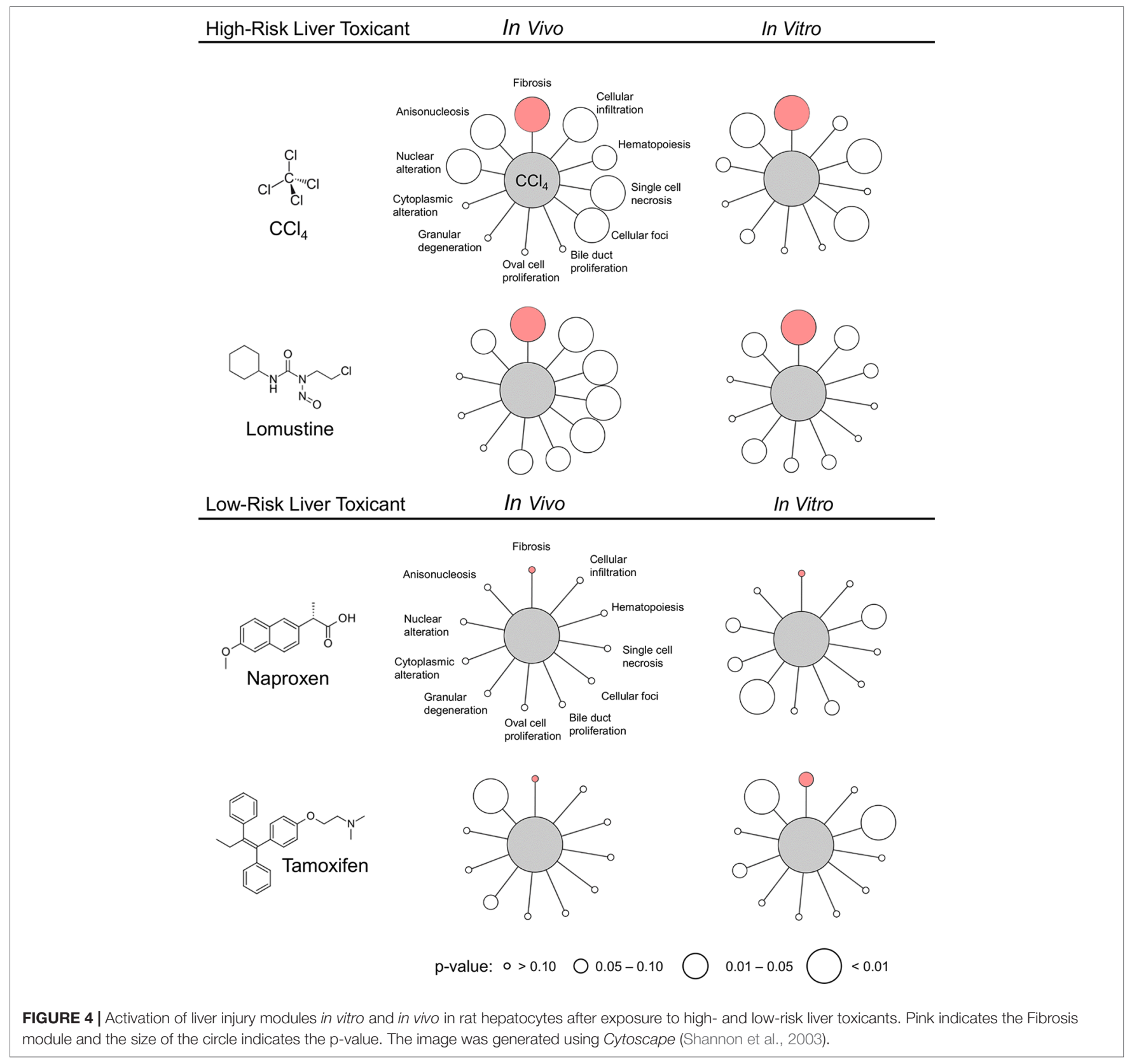

in vitro cell studies. Although this method may never replace animal studies, in conjunction with other in vitro assays, it could facilitate the screening of large numbers of chemicals in order to predict liver and kidney injuries in vivo. Consequently, the approach can reduce the number of animals needed in experiments and improve the efficiency of toxicity assessments.

\section{DATA AVAILABILITY STATEMENT}

The files from RNA-seq analysis were deposited in NCBI's Gene Expression Omnibus (GEO) database under series accession numbers GSE120195 and GSE134569.

\section{ETHICS STATEMENT}

This study was carried out in accordance with the Guide for the Care and Use of laboratory Animals of the U.S. Department of Agriculture, using protocols approved by the Vanderbilt University Institutional Animal Care and Use Committee, and USAMRDC Animal Care and Use Review Office.

\section{AUTHOR CONTRIBUTIONS}

PS, RP, MS, and AW made substantial contributions to the concept and design of the work. MS designed the protocols 
for the animal studies. SE, TO'B, and MS performed the animal studies, analyzed injury markers in blood and urine, and collected samples. RP worked on the extraction and purification of RNA. PS analyzed the gene expression data and contributed to drafting the manuscript. RP, AW, and MS contributed to revising and editing the manuscript for important intellectual content.

\section{FUNDING}

The authors were supported by the U.S. Army Medical Research and Development Command (Fort Detrick, MD), and the Defense Threat Reduction Agency grant CBCall14-CBS-05-2-0007.

\section{REFERENCES}

AbdulHameed, M. D. M., Tawa, G. J., Kumar, K., Ippolito, D. L., Lewis, J. A., Stallings, J. D., et al. (2014). Systems Level Analysis and Identification of Pathways and Networks Associated with Liver Fibrosis. PloS One 9, e112193. doi: 10.1371/journal.pone.0112193

AbdulHameed, M. D. M., Ippolito, D. L., Stallings, J. D., and Wallqvist, A. (2016). Mining kidney toxicogenomic data by using gene co-expression modules. BMC Genomics 17, 790. doi: 10.1186/s12864-016-3143-y

Ackermann, M., and Strimmer, K. (2009). A general modular framework for gene set enrichment analysis. BMC Bioinf. 10, 47. doi: 10.1186/1471-2105-10-47

Adeleye, Y., Andersen, M., Clewell, R., Davies, M., Dent, M., Edwards, S., et al. (2015). Implementing toxicity testing in the 21 st century (TT21C): Making safety decisions using toxicity pathways, and progress in a prototype risk assessment. Toxicology 332, 102-111. doi: 10.1016/j.tox.2014.02.007

Bergmann, S., Ihmels, J., and Barkai, N. (2003). Iterative signature algorithm for the analysis of large-scale gene expression data. Phys. Rev. E. 67, 031902. doi: 10.1103/PhysRevE.67.031902

Bray, N. L., Pimentel, H., Melsted, P., and Pachter, L. (2016). Near-optimal probabilistic RNA-seq quantification. Nat. Biotechnol. 34, 525. doi: 10.1038/nbt.3519

Childs, J. F. L., and Siegler, E. A. (1945). Compounds for control of orange decays. Science 102, 68. doi: 10.1126/science.102.2638.68

De Abrew, K. N., Overmann, G. J., Adams, R. L., Tiesman, J. P., Dunavent, J., Shan, Y. K., et al. (2015). A novel transcriptomics based in vitro method to compare and predict hepatotoxicity based on mode of action. Toxicology 328, 29-39. doi: 10.1016/j.tox.2014.11.008

Dix, D. J., Houck, K. A., Martin, M. T., Richard, A. M., Setzer, R. W., and Kavlock, R. J. (2007). The ToxCast program for prioritizing toxicity testing of environmental chemicals. Toxicol. Sci. 95, 5-12. doi: 10.1093/toxsci/kfl103

Dwivedi, D. K., and Jena, G. B. (2018). Glibenclamide protects against thioacetamide-induced hepatic damage in Wistar rat: investigation on NLRP3, MMP-2, and stellate cell activation. Naunyn-Schmiedeberg's Arch. Pharmacol. 391, 1257-1274. doi: 10.1007/s00210-018-1540-2

Fisher, R. A. (1932). Statistical methods for research workers (London: Oliver and Boyd).

Fitzhugh, O. G., and Nelson, A. A. (1948). Liver tumors in rats fed thiourea or thioacetamide. Science 108, 626. doi: 10.1126/science.108.2814.626

Ganter, B., Tugendreich, S., Pearson, C. I., Ayanoglu, E., Baumhueter, S., Bostian, K. A., et al. (2005). Development of a large-scale chemogenomics database to improve drug candidate selection and to understand mechanisms of chemical toxicity and action. J. Biotechnol. 119, 219-244. doi: 10.1093/nar/28.1.27

Goh, J.-Y., Weaver, R. J., Dixon, L., Platt, N. J., and Roberts, R. A. (2015). Development and use of in vitro alternatives to animal testing by the pharmaceutical industry 1980-2013. Toxicol. Res. 4, 1297-1307. doi: 10.1039/ C5TX00123D

Hajovsky, H., Hu, G., Koen, Y., Sarma, D., Cui, W., Moore, D. S., et al. (2012). Metabolism and toxicity of thioacetamide and thioacetamide S-oxide in rat hepatocytes. Chem. Res. Toxicol. 25, 1955-1963. doi: 10.1021/tx3002719

\section{ACKNOWLEDGMENTS}

The opinions and assertions contained herein are the private views of the authors and are not to be construed as official or as reflecting the views of the U.S. Army, the U.S. Department of Defense, or The Henry M. Jackson Foundation for Advancement of Military Medicine, Inc. This paper has been approved for public release with unlimited distribution.

\section{SUPPLEMENTARY MATERIAL}

The Supplementary Material for this article can be found online at: https://www.frontiersin.org/articles/10.3389/fgene.2019.01233/ full\#supplementary-material

Hamadeh, H. K., Knight, B. L., Haugen, A. C., Sieber, S., Amin, R. P., Bushel, P. R. et al. (2002). Methapyrilene toxicity: anchorage of pathologic observations to gene expression alterations. Toxicol. Pathol. 30, 470-482. doi: 10.1080/019262 30290105712

Hoofnagle, J. H., Serrano, J., Knoben, J. E., and Navarro, V. J. (2013). LiverTox: a website on drug-induced liver injury. Hepatology 57 (3), 873-874. doi: 10.1002/ hep. 26175

Igarashi, Y., Nakatsu, N., Yamashita, T., Ono, A., Ohno, Y., Urushidani, T., et al. (2015). Open TG-GATEs: a large-scale toxicogenomics database. Nucleic Acids Res. 43, D921-D927. doi: 10.1093/nar/gku955

Irizarry, R. A., Hobbs, B., Collin, F., Beazer-Barclay, Y. D., Antonellis, K. J., Scherf, U., et al. (2003). Exploration, normalization, and summaries of high density oligonucleotide array probe level data. Biostatistics 4, 249-264. doi: 10.1093/biostatistics/4.2.249

Kanehisa, M., and Goto, S. (2000). KEGG: kyoto encyclopedia of genes and genomes. Nucleic Acids Res. 28, 27-30. doi: 10.1093/nar/28.1.27

Kauffmann, A., Gentleman, R., and Huber, W. (2008). arrayQualityMetrics-a bioconductor package for quality assessment of microarray data. Bioinformatics 25, 415-416. doi: 10.1093/bioinformatics/btn647

Ledda-Columbano, G. M., Coni, P., Curto, M., Giacomini, L., Faa, G., Oliverio, S., et al. (1991). Induction of two different modes of cell death, apoptosis and necrosis, in rat liver after a single dose of thioacetamide. Am. J. Pathol. 139, 1099-1109.

Li, X., Benjamin, I. S., and Alexander, B. (2002). Reproducible production of thioacetamide-induced macronodular cirrhosis in the rat with no mortality. J. Hepatol. 36, 488-493. doi: 10.1016/S0168-8278(02)00011-9

Liberzon, A., Subramanian, A., Pinchback, R., Thorvaldsdóttir, H., Tamayo, P., and Mesirov, J. P. (2011). Molecular signatures database (MSigDB) 3.0. Bioinformatics 27, 1739-1740. doi: 10.1093/bioinformatics/btr260

Minowa, Y., Kondo, C., Uehara, T., Morikawa, Y., Okuno, Y., Nakatsu, N., et al. (2012). Toxicogenomic multigene biomarker for predicting the future onset of proximal tubular injury in rats. Toxicology 297, 47-56. doi: 10.1016/j. tox.2012.03.014

Okuyama, H., Nakamura, H., Shimahara, Y., Uyama, N., Kwon, Y.-W., Kawada, N., et al. (2005). Overexpression of thioredoxin prevents thioacetamide-induced hepatic fibrosis in mice. J. Hepatol. 42, 117-123. doi: 10.1016/j.jhep.2004.09.020

Parmentier, C., Couttet, P., Wolf, A., Zaccharias, T., Heyd, B., Bachellier, P., et al. (2017). Evaluation of transcriptomic signature as a valuable tool to study druginduced cholestasis in primary human hepatocytes. Arch. Toxicol. 91, 28792893. doi: 10.1007/s00204-017-1930-0

Pimentel, H., Bray, N. L., Puente, S., Melsted, P., and Pachter, L. (2017). Differential analysis of RNA-seq incorporating quantification uncertainty. Nat. Methods 14, 687. doi: 10.1038/nmeth.4324

Pontes, B., Giráldez, R., and Aguilar-Ruiz, J. S. (2015). Biclustering on expression data: a review. J. Biomed. Inf. 57, 163-180. doi: 10.1016/j.jbi.2015.06.028

Sahini, N., Selvaraj, S., and Borlak, J. (2014). Whole genome transcript profiling of drug induced steatosis in rats reveals a gene signature predictive of outcome. PloS One 9, e114085. doi: 10.1371/journal.pone.0114085 
Schyman, P., Printz, R. L., Estes, S. K., Boyd, K. L., Shiota, M., and Wallqvist, A. (2018). Identification of the toxicity pathways associated with thioacetamideinduced injuries in rat liver and kidney. Front. Pharmacol. 9, 1272. doi: 10.3389/ fphar.2018.01272

Segal, E., Friedman, N., Koller, D., and Regev, A. (2004). A module map showing conditional activity of expression modules in cancer. Nat. Genet. 36, 1090. doi: $10.1038 / \mathrm{ng} 1434$

Shannon, P., Markiel, A., Ozier, O., Baliga, N. S., Wang, J. T., Ramage, D., et al. (2003). Cytoscape: a software environment for integrated models of biomolecular interaction networks. Genome Res. 13, 2498-2504. doi: 10.1101/gr.1239303

Steiner, G., Suter, L., Boess, F., Gasser, R., de Vera, M. C., Albertini, S., et al. (2004). Discriminating different classes of toxicants by transcript profiling. Environ. Health Perspect. 112, 1236-1248. doi: 10.1289/txg.7036

Sutherland, J. J., Jolly, R. A., Goldstein, K. M., and Stevens, J. L. (2016). Assessing concordance of drug-induced transcriptional response in rodent liver and cultured hepatocytes. PloS Comp. Biol. 12, e1004847. doi: 10.1371/journal.pcbi.1004847

Sutherland, J. J., Webster, Y. W., Willy, J. A., Searfoss, G. H., Goldstein, K. M., Irizarry, A. R., et al. (2017). Toxicogenomic module associations with pathogenesis: a network-based approach to understanding drug toxicity. Pharmacogenom. J. 18, 377. doi: 10.1038/tpj.2017.17

Sutherland, J. J., Stevens, J. L., Johnson, K., Elango, N., Webster, Y. W., Mills, B. J., et al. (2019). A novel open access web portal for integrating mechanistic and toxicogenomic study results. Toxicol. Sci. 170, 296-309. doi: 10.1093/toxsci/ $\mathrm{kfz} 101$

Taškova, K., Fontaine, J.-F., Mrowka, R., and Andrade-Navarro, M. A. (2018). Evaluation of in vivo and in vitro models of toxicity by comparison of toxicogenomics data with the literature. Methods 132, 57-65. doi: 10.1016/j.ymeth.2017.07.010

Tawa, G. J., AbdulHameed, M. D. M., Yu, X., Kumar, K., Ippolito, D. L., Lewis, J. A., et al. (2014). Characterization of chemically induced liver injuries using gene co-expression modules. PloS One 9, e107230. doi: 10.1371/journal.pone. 0107230

Te, J. A., AbdulHameed, M. D. M., and Wallqvist, A. (2016). Systems toxicology of chemically induced liver and kidney injuries: histopathology-associated gene co-expression modules. J. Appl. Toxicol. 36, 1137-1149. doi: 10.1002/jat.3278
Van den Hof, W. F. P. M., Coonen, M. L. J., van Herwijnen, M., Brauers, K., Jennen, D., Olde Damink, S. W. M., et al. (2017). Validation of gene expression profiles from cholestatic hepatotoxicants in vitro against human in vivo cholestasis. Toxicol. In Vitro 44, 322-329. doi: 10.1016/j.tiv.2017.07.024

Wetmore, B. A. (2015). Quantitative in vitro-to-in vivo extrapolation in a highthroughput environment. Toxicology 332, 94-101. doi: 10.1016/j.tox.2014.05.012

Wu, G., Fang, Y.-Z., Yang, S., Lupton, J. R., and Turner, N. D. (2004). Glutathione metabolism and its implications for health. J. Nutr. 134, 489-492. doi: 10.1093/ jn/134.3.489

Yeh, C.-N., Maitra, A., Lee, K.-F., Jan, Y.-Y., and Chen, M.-F. (2004). Thioacetamideinduced intestinal-type cholangiocarcinoma in rat: an animal model recapitulating the multi-stage progression of human cholangiocarcinoma. Carcinogenesis 25, 631-636. doi: 10.1093/carcin/bgh037

Yu, C., Woo, H. J., Yu, X., Oyama, T., Wallqvist, A., and Reifman, J. (2017). A strategy for evaluating pathway analysis methods. BMC Bioinf. 18, 453. doi: 10.1186/s12859-017-1866-7

Zerbino, D. R., Achuthan, P., Akanni, W., Amode, M. R., Barrell, D., Bhai, J., et al. (2018). Ensembl 2018. Nucleic Acids Res. 46, D754-D761. doi: 10.1093/nar/ gkx1098

Zhang, J. D., Berntenis, N., Roth, A., and Ebeling, M. (2013). Data mining reveals a network of early-response genes as a consensus signature of drug-induced in vitro and in vivo toxicity. Pharmacogenom. J. 14, 208. doi: 10.1038/tpj.2013.39

Conflict of Interest: The authors declare that the research was conducted in the absence of any commercial or financial relationships that could be construed as a potential conflict of interest.

Copyright (c) 2019 Schyman, Printz, Estes, O'Brien, Shiota and Wallqvist. This is an open-access article distributed under the terms of the Creative Commons Attribution License (CC BY). The use, distribution or reproduction in other forums is permitted, provided the original author(s) and the copyright owner(s) are credited and that the original publication in this journal is cited, in accordance with accepted academic practice. No use, distribution or reproduction is permitted which does not comply with these terms. 